\title{
Data Network Simulator with Classical Ballet
}

Genevieve Smith-Nunes

\author{
ReadySaltedCode CIC \\ 1,3 Knoyle Rd \\ Brighton
}

Genevieve@readysaltedcode.org
Peter Cook

Animated Data Ltd

The Skiff

Brighton
Camilla Neale

University of Worcester

Henwick Grove

Worcester WR2 6AJ, UK

c.neale@worc.ac.uk
Paul Golz

University of Worcester

Henwick Grove

Worcester WR2 6AJ, UK

p.golz@worc.ac.uk

[data]storm, from readysaltedcode CIC, a data driven dance performance. The development of a social network simulator to demonstrate network growth and message propagation. The underpinning theory of piece stems from social network theory (SNT), graph theory, computer mediated communication (CMC) through to social information processing (SIP) and Computational Thinking (CT). The data visualisation is linked to the physical ballet movements of the dancers, they are a manifestation of the data. The data visualisations on screen link to the live dancers performance patterns and modify to create the visuals and movements of data transmission across a network.

Network growth. The first of the simulations shows network growth. Each node in the network represents a user who has the following characteristics:

- friendliness (how often they're likely to make friends with another user)

- $\quad$ chattiness (how often they send out messages)

- $\quad$ category (the subject area they're most interested in)

At random time intervals things occur: New users are added to the network depending on the above characteristics, users become friends with each other. All the rules stay the same throughout the simulation.

At the same time the dance (ballet) movements and wearables (LEDS) were choreographed/coded to accompany the data visualisation using network mapping techniques. The choreography and wearables elements link to the friendliness and chattiness of each of the nodes in the simulated network. This network simulation is further utilised in the Virus section of the performance using the same rules to simulate how a virus can spread through a network. Further work on this simulation will look at two things 1 . Message propagation and viral messaging within a social network like Twitter. 2. Pain signals within the body and how they compare to data transfer within a social network. 


\section{INTRODUCTION}

This paper is focusing on the two data visualisations of the second ballet, [data]Storm. The subject for this performance is linking weather data with networks and data transmission. The two data visualisations were used for the Networks and Virus pieces in the ballet.

The Art of Computer Science is a series of data driven classical ballets using the theories and concepts of Computer Science, Using Biomedical engineering and data as the story telling with the dancers acts as data visualisation. The data, choreography, visuals and wearables of each ballet are treated equally throughout the entire project. (Golz, Smith-Nunes 2015)

The project was born from Genevieve SmithNunes' Computing teaching experience over the past ten years. The changes and challenges engaging girls into Computer Science with a focus on 'STEAM' (Science, Technology, Engineering, Arts and Math). The concept had arisen from the new Computing curriculum back in 2012, which is now compulsory in schools as of September 2014. Each ballet provides a cross-disciplinary approach to learning, using the physical medium of dance to engage learners in complex theories in computer science. The on-going project currently has three ballets: [arra]Stre, [data]Storm and [pain]Byte. All are data driven performances that open up the creative opportunities of digital, and explores how data visualisations, wearables and dance can untangle and clarify difficult concepts.

The computer science concept and theories used for the educational side of the project were taken from the A Level Computing OCR exam board specifications, England. The concepts selected for the project came from the following sub sections Data Structures and Implications of Computer Use in the exam board specification. They require students to understand data structures, data types, data transmission, network structures and protocols. The second section focuses on the implications of computer use both legal and social. (OCR.org). The theory for the web based network and virus simulations and associated choreography come from social network theory (SNT) and social information processing (SIP).

The partnership and collaboration is between readysaltedcode CIC, Animated Data Ltd, Glaston Bridge Software, Choreographers from the University of Worcester and secondary aged Students from Battle Abbey School. The students are also the dancers who contribute and participate in the development of the choreography and wearables. We have seen direct impact on the students and teachers in their confidence within this subject area from participating in this program.

The ongoing project has received several awards including an International Google RISE award for Computer Science Education and two Arts Council England's, Grants for the Arts.

\section{THEORY}

For the network simulations referred to in this paper we focused on the following theories (though other theories were used in the project as a whole). Social network theory (SNT) and social information processing theory (SIP)

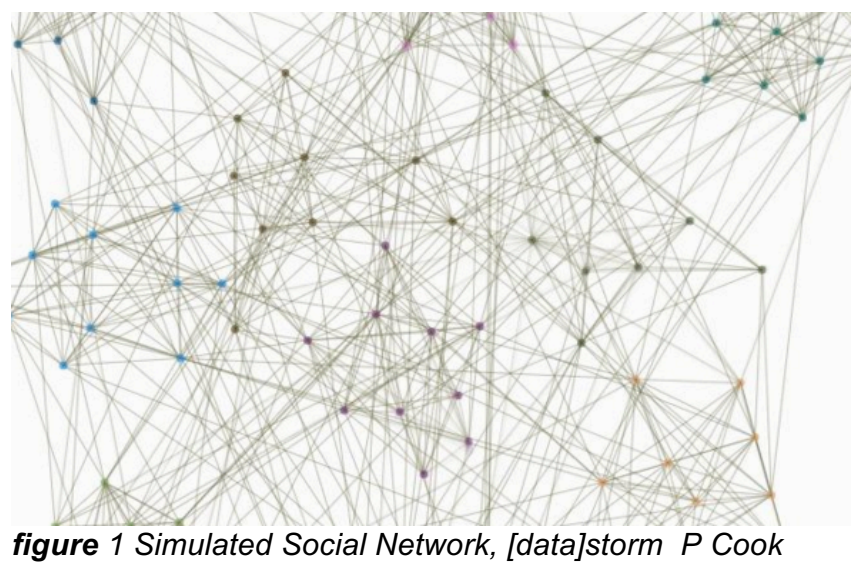

figure 1 Simulated Social Network, [data]storm P Cook

A social network is a network made up of individuals or groups which are the nodes. Each node is connected to others nodes by different characteristics such as friendship, common interests, location or other dependencies. With this we used SNT to create the rules for the simulations. The network as the simulation of the interactions and the patterns created in a simple simulated social network. The characteristic of that of the friends (nodes) are connected to other nodes (friends of friends) and their clustering coefficient, how they tend to group together forming "smallworld networks" (Watts and Strogatz, 1998). The structure and connections of the network simulations enabled the development of the dance choreography as a representation of the nodes, connections and data transfer out of the the theoretical model / simulated network.

Social Information processing theory (SIP) played an important part role in the continuous asynchronous development of the piece. SIP is an interpersonal communication theory developed by Joseph Walther in 1992. This theory explain how people get to know one another online (without non-verbal clues) and they develop and manage 
relationships in a computer mediated environment (CME).

However, online interpersonal relationships in a CME may require more time to develop than traditional face to face relationships. An important point is that established, online personal relationships may demonstrate the same relational dimensions and qualities as a face to face relationships. The online relationship may help facilitate connections that would not have formed in the face to face world due to intergroup difference, geographical challenges for example.

There are many theories relating to computer mediated communication (CMC). Prior to SIP there were two relevant theories social presence theory (SPT) and media richness theory (MRT). These two theories differ from CMC in that they concluded CMC was NOT a good medium for developing relationships. SIP challenges the ideas in both SPT and MRT. New theories have developed an increasingly optimistic view of online communication, explaining how those who interact online are highly capable of creating and maintaining impressions and connections with others online.

Further research led Walther to "Hyperpersonal Model theory" which demonstrates that in certain circumstances CMC is even better at developing the depth of affection and emotion than similar situations of face to face interpersonal communication. This model also offers a strong view of CMC. Important factors include:

- sender

- receiver

- channel

- feedback

Asynchronous communication i.e email is sent but received at different times. This could cause problems for both interpersonal relationships in CMC. However according to this model, asynchronous communication allows both optimised self presentation by the message sender and idealised perceptions of the sender by the message receivers.

\section{VISUALISATIONS - SIMULATED NETWORKS}

Based on the theory above we implemented two simulations: a social network simulator and a virus simulator. The former simulates the growth of a network based on each actor's (or node's) friendliness, chattiness and interests. The latter, built on top of the social network simulator, simulates the propagation of messages through the network. On one level this can be thought of a messaging system, but we believe that viruses, be they computer or biological, spread in a similar manner. It's no coincidence that the term 'go viral' is used to describe messages and videos that have suddenly become extremely popular on social networks.

The simulators were built using web-standards HTML, SVG, CSS and JavaScript and shared on the Codepen platform (Figure 1). This allows the simulators to be freely shared and modified.

\subsection{Social Network Simulator}

The social network simulator aims to demonstrate the growth of a social network based on a few simple rules. Each node in the network represents a user who has the following characteristics:

- friendliness (how often they're likely to make friends with another user)

- chattiness (how often they send out messages)

- category (the subject area they're most interested in)

The terms were taken from traditional sociological social network theory to aid understanding for the participants involved (Using Milgram's original work in 1967 through to more recent, mathematical, research from Valente. T, Palinkas. L, Czaja.S, Kar-Hai. C and Brown. CH 2015 on Social Network Analysis for Program Implementation). The rules enable the importance of the relationships to be highlighted and then generated as seen in (SNT).

Friendliness indicates how likely they are to make friends with another user whilst chattiness indicates how likely they are to be noticed by other users. The category indicates which subject (in our case, this is represented by a number between 1 and 10) the user is most interested in. A user is most likely to connect with users who share the same interests.

At random time intervals two things occur:

- new users are added to the network

- depending on the above characteristics, users become friends with each other 


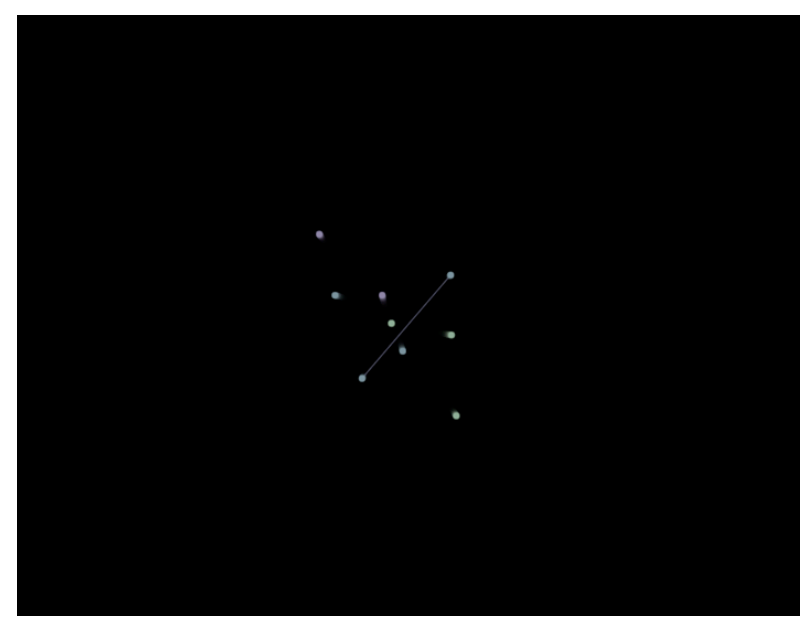

Figure 2a Early stages of the social network

All the rules stay the same throughout the simulation. When we run it, we see the network start with just a few scattered nodes (Figure 2a). It then starts to take shape, with users tending to congregate in groups with the same interest (Figure $2 b)$. As the simulation continues, more and more connections are added, and eventually we get a very dense connected network. As seen in figures $2 b$ \& 4 .

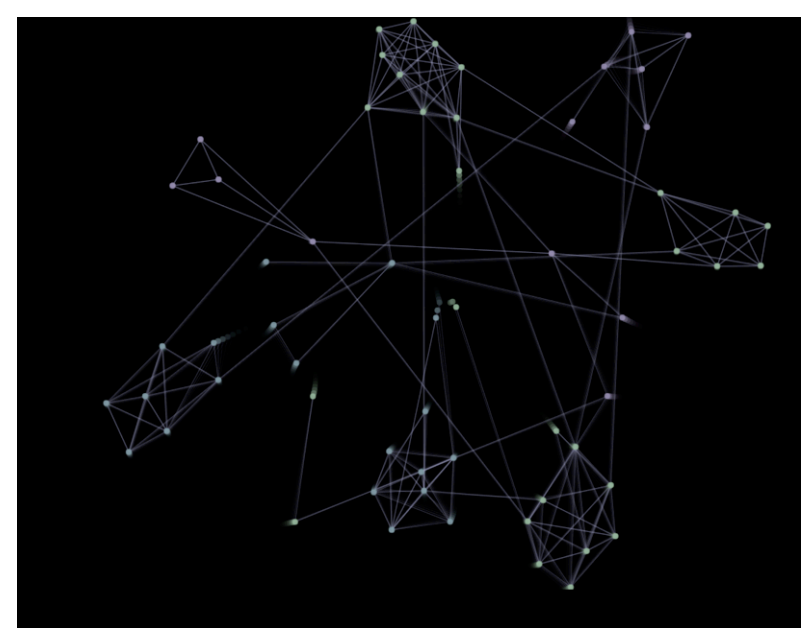

Figure $2 b)$ after a few iterations nodes have started forming into groups sharing the same interest

\subsection{Virus Simulation}

The virus simulation shows how messages spread through the network. Every now and then, a user will create a message. A user sending out a message is represented by the user becoming a coloured dot. The user's friends will be able to see the message, and depending on how interesting the message is, the friend may choose to repeat the message. (In Twitter terms, this is a retweet.)
When the simulation runs the network slowly grows and every now and then a user messages. Occasionally a friend will repeat the message. As the network density grows, we tend to see messages getting repeated within the same niche (i.e. group of users who share the same category) but before long, the messages go viral and spread throughout the whole network (Figure 3). The changes happened as the code rules allowed the virus (turning each node green) to spread through the network.

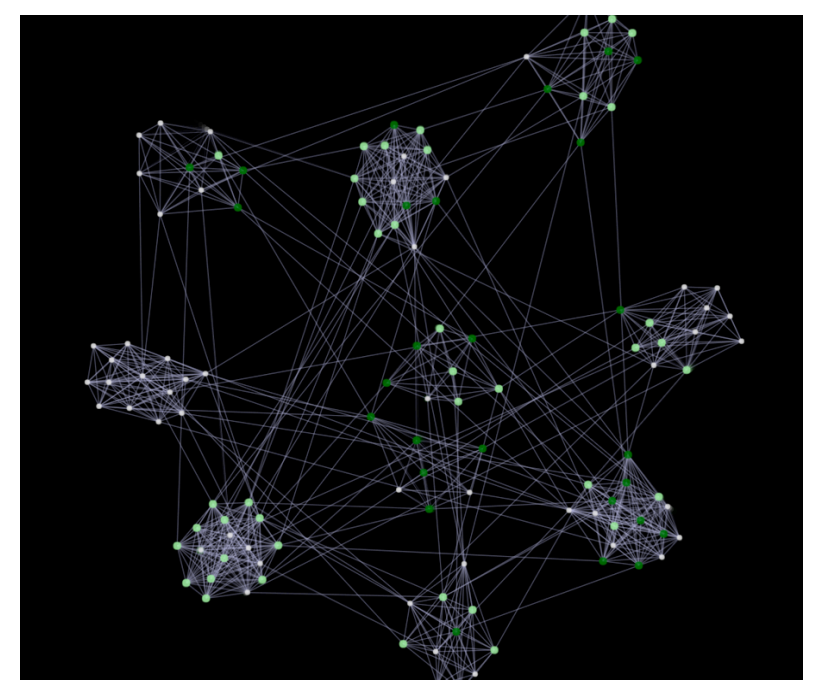

Figure 3. Messages going viral, indicated by the green coloured dots

We found with both simulations that with a few simple rules, we successfully simulated social network growth and viral messaging.

\section{CHOREOGRAPHY}

The virus was choreographed to sit within the data visualisations. At the time of the virus's entrance the data forms together to create multiple circular mass which indicate the initial problem. At this point we felt the choreography-needed to be aggressive and fast to assist the visual. The idea being that the virus over-ruled the other data so cleared the stage in an attack of grand jete's which was initiated by the change of colour on set. The numerous pose turns and fouette pirouettes indicate confusion within the programme and mirrored the movements of the virus simulation.

The choreography for each individual dancer has the same entrance and exit motifs which relate to the data in its original unaffected state, (not yet affected by the virus). Each dancer's choreography (data) then diverges depending on the effect of the virus. Using example viruses such as the Xerox 
worm program the choreography demonstrates the ability to propagate through a network. The work "Malware" of digital artist Alex Dragulescu (Dragulescu, A. 2011), informed the shapes of the choreography linked to the progression of the data visualisation.

Using social networks familiar to the students we then looked at a paper on "Towards a Theory of Trust in Networks of Humans and Computers" (Gligor and Wing 2008). Specifically, section 7.3 Promoting Cooperation in Networks of Humans and Computers. The idea that high levels of trust beget high levels of trust. If a social network has some of elements of this does this allow a virus (sender betrayal) to propagate the network in the same way? This idea was use to the development of the virus choreography for each of the dancers representing the data bits.

The formal language of classical ballet, with set structures, routines, rules and terminology, can be married with that of social network theory, programming and computational thinking. It is because of this association that classical ballet was selected as the dance medium over other forms of dance.

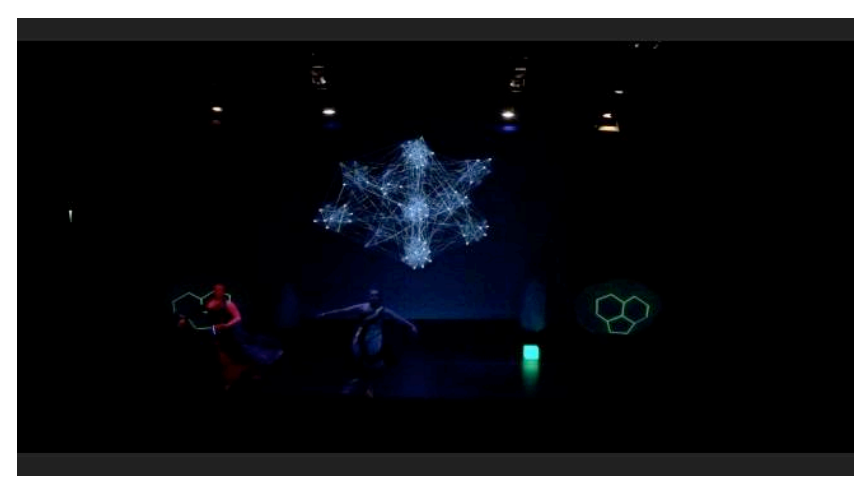

Figure47: Virus - live performance

Figure4 show the virus towards the end of the dance section. The image shows a single glowing cube which represents a single bit of data travelling through a network to arrive at its destination. The cube is blue excepts for the virus section. Each dancer represented a node and their choreography was altered by the introduction of the virus. The dancer themselves generated the choreography based on data visualisation of various virus.

\section{COMPUTATIONAL THINKING}

The link between the computing education and data visualisation was clear in the process of developing the choreography as mentioned in the previous section. The use of the Dargulescu's work was used to enable the students to use Computational Thinking (Wing 2006) as a process throughout the development of the choreography. The students used both the network and virus simulations as the basis for their individual movements and motif. The initial abstraction gained through the imagery which enable the students to offer generalisations. The visualisations enabled the students to effectually use computational thinking in developing their individual choreography. The characteristics of computational thinking apply to all subjects not just computer science. A simplified version of the characteristics:

- Abstraction (removing unnecessary detail)

- Logic (prediction and analysing)

- Patterns (spotting and using similarities)

- Decomposition (breaking down into parts)

- Algorithms (making steps and rules)

- Evaluation (making judgement)

The visualisations Network and Virus were designed to work both with and without the ballet dancers. The impact of data transmission and node maps conceptual link is slightly less without the dancers for a theatre audience. A classroom audience is able to see networks generate in real time alongside the code. This enables a 'classroom' audience to visualise and abstract the nature of the transmission of data within the simulated network. This will be discussed further in another paper.

\section{FUTURE}

[pain]Byte is the next stage of the project which will looking at chronic pain and biomedical engineering. This data driven ballet will further develop the network visualisation linking pain signals to those of the simulated social network used in [data]Storm. Using medical studies such as the research on the 'Sustained Effectiveness of $10 \mathrm{kHz}$ High-Frequency Spinal Cord Stimulation for Patients with Chronic, Low Back Pain' (Al-Kaisy, A., Van Buyten, J.-P., Smet, I., Palmisani, S., Pang, D. and Smith, T. (2014)). Information and raw data will be generated by the dancers using wearables sensor built in into the costumes. Data will be transmitted for use in the data visualisations on screen both in the live performance and online. The storytelling of the piece is about the rise of technology in the field of health and the positive impacts that this has on chronic pain sufferers. Data visualisations will be use to manifest the invisible nature of what it is to be in chronic pain and how pain signals are transmitted. 


\section{CLOSING REMARKS}

There are two strands to this paper that can be studied and used together or independently. One is data visualisation and simulation to enhance the understanding of social networking. Two is choreographed ballet movements to explain a variety of relationships and social interactions within these networks. Future studies should be directed at

- understanding the methods and speed of these forms of communication

- Identifying factors in the form and content of the most desirable / popular messages

- Predicting outcomes for both producers and consumers of the ideas

\section{REFERENCES}

\section{Journal article:}

Sih, A., Hanser, S. and McHugh, K. (2009). Social network theory: new insights and issues for behavioral ecologists. Behavioral Ecology and Sociobiology, 63(7), pp.975-988.

Tidwell, L.C. and Walther, J.B., 2002. Computer-mediated communication effects on disclosure, impressions, and interpersonal evaluations: Getting to know one another a bit at a time. Human communication research, 28(3), pp.317-348.

Walther, J.B., 2007. Selective self-presentation in computer-mediated communication: Hyperpersonal dimensions of technology, language, and cognition. Computers in Human Behavior, 23(5), pp.25382557.

Milligram S (May, 1967)The Small-World Problem Psychology Today, vol. 1, no. 1, pp61-67

Denefrio, Lauren A; Brandy Russell; David Lopatto; and Yi Lu. 2007. "Linking Student Interests to Science Curricula," Science, 318: 1872-1873

J.M. Wing, "Computational Thinking," CACM, viewpoint, vol. 49, no.3 March 2006, pp. 33-35

Valente TW, Palinkas LA, Czaja S, Chu K-H, Brown CH (2015) Social Network Analysis for Program Implementation. PLoS ONE 10(6): e0131712. doi:10.1371/journal.pone.0131712

\section{Book}

P. W. Holland and S. Leinhardt (1971). "Transitivity in structural models of small groups". Comparative Group Studies 2: 107-124.

Mitchell, William J. "Boundaries/Networks." In Me++: The Cyborg Self and the Networked City. Cambridge, MA: MIT Press, 2003. pp. 7-17. ISBN: 9780262134347.

\section{Chapter}

Kadushin, C (2014) Chapter 2. Some Basic Network Concepts and Propositions, Introduction to Social Network Theory,

\section{Conference}

Opsahl T(2009). "Clustering in Two-mode Networks". Conference and Workshop on TwoMode Social Analysis (Sept 30-Oct 2, 2009)

Golz, P. and Smith-Nunes, G., 2015, July. [arra] stre: a data-driven ballet. In Proceedings of the Conference on Electronic Visualisation and the Arts (pp. 90-91). British Computer Society. 
Tsan, J., Boyer, K.E. and Lynch, C.F., 2016, February. How Early Does the CS Gender Gap Emerge?: A Study of Collaborative Problem Solving in 5th Grade Computer Science. In Proceedings of the 47th ACM Technical Symposium on Computing Science Education (pp. 388-393). ACM.

V. Gligor and J.M. Wing " Towards a Theory of Trust in Networks of Humans and Computers," in Proceedings of Nineteenth International Workshop on Security Protocols, Cambridge, England, March 28-30, 2011, Invited Paper.

\section{Online}

Gnu.org, (2016). The GNU General Public License v3.0 - GNU Project - Free Software Foundation. [online] Available at: http://www.gnu.org/licenses/gpl-3.0.en.html

[Accessed 23 Feb. 2016].

Ocr.org.uk, (2016). [online] Available at: http://www.ocr.org.uk/lmages/70162-

specification.pdf [Accessed 23 Feb. 2016].

Mensvoort, K. (2008). Meet the Worms, Viruses and Trojans | Next Nature Network. [online] Next Nature Network. Available at: https://www.nextnature.net/2008/02/meet-theworms-viruses-and-trojans/ [Accessed 08 Jan. 2015].

Dspace.mit.edu, (2004). 21A.350J / SP.484J / STS.086J / WGS.484J The Anthropology of Computing, Fall 2004.

Al-Kaisy, A., Van Buyten, J.-P., Smet, I., Palmisani, S., Pang, D. and Smith, T. (2014), Sustained Effectiveness of $10 \mathrm{kHz}$ High-Frequency Spinal Cord Stimulation for Patients with Chronic, Low Back Pain: 24-Month Results of a Prospective Multicenter Study. Pain Medicine, 15: 347-354. doi: 10.1111/pme.12294

Brain networks: small-worlds, after all? lopscience.iop.org, (2016). [online] Available at: http://iopscience.iop.org/article/10.1088/1367-

2630/10/8/083042/pdf [Accessed 11 Dec. 2015].

Sq.ro, (2011). Alex Dragulescu - works catalog. [online] Available at: https://sq.ro/ [Accessed 1 June. 2015]. 